\title{
Chemical Surface Analysis with Nanometer Depth Resolution
}

\author{
Roland Hauert* and Beat A. Keller
}

\begin{abstract}
The characterization of surface chemical species with respect to their identity and quantification becomes a very demanding task in the case of submicron or nanoscale materials and systems. In such cases, the major part of atoms or molecules within a typical analytical volume of about $10^{-12} \mathrm{~cm}^{3}$ must be considered as surface related. Although sometimes pushed to the current instrumental limit, the use of the three most important surface analysis techniques X-ray photoelectron spectroscopy (XPS), Auger electron spectroscopy (AES), and time-of-flight secondary ion mass spectrometry (ToF-SIMS) can provide the scientific community with the necessary analytical tools to identify and quantify chemical entities on the surface of nanoscale objects.
\end{abstract}

Keywords: Auger electron spectroscopy (AES) · Chemical surface analysis · ESCA · SAM · ToF-SIMS . $X$-ray photoelectron spectroscopy (XPS)

\section{Introduction}

The ability to analyze the chemical structure and composition of solid surfaces on a length scale of nanometers is a key technology for systems development with nanometer-sized dimensions. The term nanometer depth resolution will be used in this contribution to describe analytical tools which can provide detailed chemical information located at the topmost surface layers of a material. The characterization of surface and interface chemistry has a tremendous influence on the behavior of modern materials and consequently has been in the focus of recent developments of surface analytical instrumentation.

We aim, in this paper, to describe the implications of state-of-the-art equipment on research and development of modern materials. However, understanding the chemistry of a system at the surface with all the interactions and forces acting across interfaces means also to describe and locate pos-

\footnotetext{
${ }^{\star}$ Correspondence: Dr. R. Hauert

Swiss Federal Laboratories for Materials Testing and

Research, Empa

Ueberlandstr. 129

$\mathrm{CH}-8600$ Dübendorf

Tel.: +41448234558

E-Mail: roland.hauert@empa.ch
}

sible failure mechanisms at the nanoscale. In order to obtain a detailed picture of such phenomena, it is vital to have access to the most important instruments and technologies available today. Although a range of very sophisticated methods has been developed for special problems arising in interface chemical analysis, the most important methods still remain X-ray photoelectron spectroscopy (XPS), Auger electron spectroscopy (AES), and time-of-flight secondary ion mass spectrometry (ToF-SIMS). The availability of modern equipment to perform analytical investigation using these methods allows the basic analytical questions to be answered: "what is it; where is it localized; and how much of it is present?" As simple as these questions look in the light of today's widely used trace analysis capabilities, the application to surface characterization still requires advanced and expensive instrumental technology.

\subsection{Nanoanalysis at the Surface}

Many chemically relevant processes like adhesion, catalysis or biorecognition occur directly at the surface. This situation is briefly outlined in Fig. 1. Although many chemically and physically important phenomena have their origin some nanometers in the depth of the bulk, only the topmost few layers of a material can really be termed as surface related. In most cases, there is a striking difference between the chemical composition of a bulk material and the entities located on the surface. In the worst case, the desired functionality is masked or negatively influenced because of unwanted adsorption of contaminants. Therefore, the chemical and physical properties of the surface determine later performance of a system for a given application. Since the number of bulk atoms in the detection volume is at least a few orders of magnitude higher than the surface species $\left(10^{15}\right.$ atoms $\left./ \mathrm{cm}^{2}\right)$, the available instrumental detection sensitivity of surface analytical methods has also to meet the required performance. However, for very small systems like nanoparticles, a large fraction of the atoms or molecules of the entire object are surface or near surface species. Here, one might draw a distinction between microanalysis and nanoanalysis. Even the analytical techniques with the coarsest special confinement are capable of detecting material within a domain with linear dimensions in the micrometer range, i.e. a volume of around $10^{-12} \mathrm{~cm}^{3}$. Depending on its density, the amount of material found in this volume adds up to about 1-10 pg of mass, which implies that the detection sensitivity of the applied technique must necessarily have trace analysis capabilities. In the context of surface materials characterization, this means that major constituents $(>10 \%)$, minor constituents (1 to $\leq 10 \%$ ), and trace components of the surface layer $(<1 \%)$ should clearly be distinguished. Therefore, the dynamic range of the analytical instrumentation must reflect these requirements, which in some cases reach the parts per million or parts per billion level.

All techniques presented within the scope of this paper are based on the same underlying concept of excitation of the 


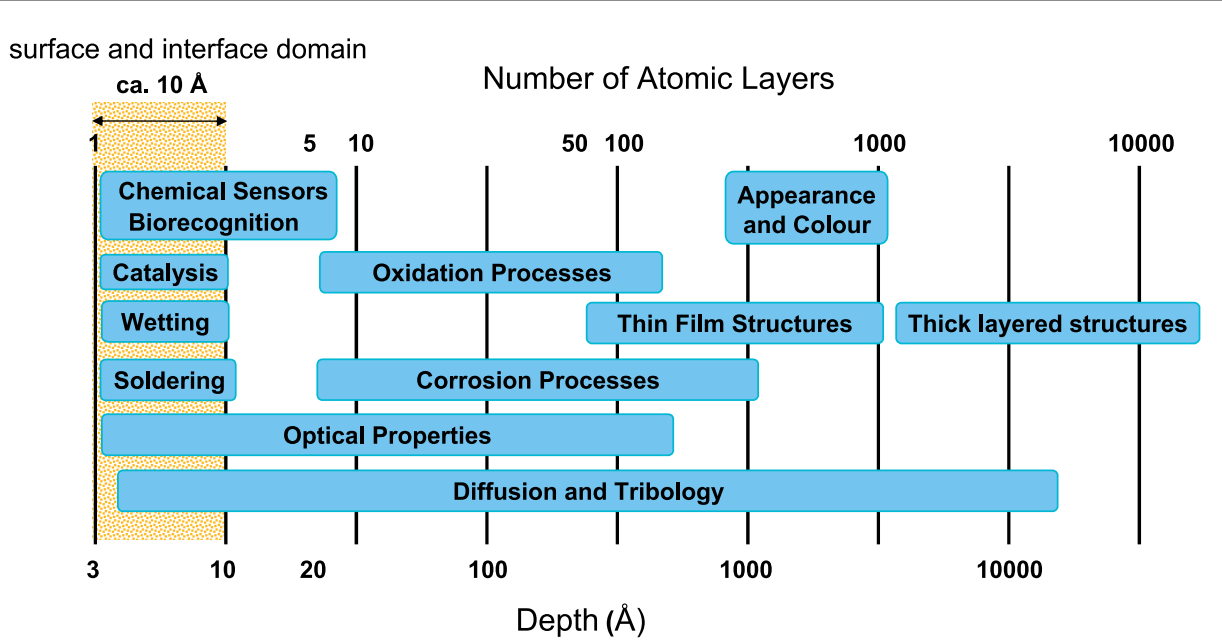

Fig. 1. Different surface effects and the surface dimensions involved

specimen with an incident energetic beam and the subsequent production and detection of characteristic species that allows identification of the chemical materials located within the analytically relevant surface area.

However, as will be discussed, there is often a striking trade-off between analytical sensitivity and the spatial or depth resolution because of increasing materials consumption and for trace analysis. A considerable degree of surface destruction might arise from such conditions, which can be a major obstacle for later analysis using techniques that are particularly sensitive to such effects, e.g. atomic force microscopy.

The scope of this presentation will be to provide the reader with an understanding of the analytical capabilities of the most important techniques used today to characterize the chemical state of a surface. First, we will provide an introduction to Xray photoelectron spectroscopy (XPS) and Auger electron spectroscopy (AES). Both methods are based on the characterization of chemical materials by analyzing the energetic state of electrons produced after the interaction with an exciting beam of radiation or particles. As will be presented, the major advantage of these methods is the exact quantification of species present at a surface. In contrast to XPS and AES, secondary molecular fragments are analyzed in time-of-flight secondary mass spectrometry (ToF-SIMS) as a result of the interaction of charged high-energy primary ions with the surface. The identification of complex molecularly organized surface layers is considered as the most important feature of this technique.

\section{Techniques and Applications}

In the following, the most relevant analytical tools for chemical surface analysis with nanometer depth resolution are described, including some surface analytical results for illustration.

\section{1. $X P S$}

XPS is a quantitative spectroscopic technique that measures the elemental composition, the chemical state and the electronic state of the elements in the first few nanometers of a surface. XPS is also known as ESCA, an abbreviation for electron spectroscopy for chemical analysis.

In an XPS analytical instrument, the surface is exposed to mono energetic $\mathrm{X}$ rays, usually $\mathrm{Mg}$ or $\mathrm{Al} \mathrm{K} \alpha$ radiation. The energy of these X-rays is elastically (without any energy loss) transferred to the electrons of the atoms present. After they overcome their binding energy, these electrons will escape from the surface where their energy is determined in an electron energy analyzer. The spectrum obtained is the number of electrons detected as a function of the binding energy the electrons had in the bulk. The surface sensitivity of $1-5 \mathrm{~nm}$ is dependent on the material analyzed and the kinetic energies of the electrons and is caused by the short mean free path of the electrons in a solid. All elements having core electrons can be detected, which means that all elements can be measured except hydrogen and helium. The detection limit is, depending on the elements and the cross section of the different core electrons, between 0.5 and 0.1 atomic percent. Since the number of core electrons is not influenced by any change in chemical state, the atomic concentrations are obtained quantitatively with an accuracy of about $10 \%$. The chemical state or the valence of the elements analyzed can be determined by the so-called chemical shift of the binding energy position of the photoelectron signals measured. To further decrease or increase the surface sensitivity, angle resolved spectra can be taken. There, the escaping electrons are collected from perpendicular to nearly parallel to the surface, resulting in a variation of the depth measured from five down to one nanometer. By ion etching with high energy argon ions, material can also be removed from the surface to obtain the elemental concentration as a function of the distance to the surface. The lateral resolution depends on the type of the different commercial instruments and is in the range of $3-30 \mu \mathrm{m}$. By using a synchrotron for $\mathrm{X}$-ray excitation, a lateral resolution of $120 \mathrm{~nm}$ [1] or even lower can be obtained. All ultrahigh vacuum compatible materials can be analyzed. An extensive introduction to surface analysis, inclusive XPS and AES can be found in [2]. Below, some applications of XPS are presented to visualize the possibilities of this analytical technique.

\subsubsection{Determination of the Silicon Dioxide Layer Thickness}

Thin $\mathrm{SiO}_{2}$ layers are used as an ultrathin gate oxide in semiconductor fabrication and are also a good system to demonstrate the possibilities of XPS to analyze thin oxide layers that are present an all metals exposed to air (except on some noble metals). The surface oxides of metallic materials determine effects like adhesion, surface energy, wetting angle as well as the biological reactions on a metallic implant. To determine the thickness of the oxide layer, the effective attenuation length, EAL, of the $\mathrm{Si} 2 \mathrm{p}$ photoelectrons in the oxide has to be known as well as the ratio of the XPS intensity of pure $\mathrm{Si}$ and pure $\mathrm{SiO}_{2}$. The electron escape angle $\Theta$ was $45^{\circ}$.

The EAL of $3.49 \mathrm{~nm}$ was taken from [3] and the ratio of the $\mathrm{Si} 2 \mathrm{p}$ signal of pure $\mathrm{SiO}_{2}$ and pure $\mathrm{Si}$ was measured separately. To determine the oxide layer thickness the formula below was used, for details see ref. [4]. To measure the oxide layer thickness of the different silicon oxides, the $\mathrm{Si} 2 \mathrm{p}$ signal was measured on all samples and is displayed in Fig. 2. For data analysis, the spectra have been fitted with three peaks, two for the elemental $\mathrm{Si}$ with a fixed spin orbit splitting of $0.6 \mathrm{eV}$ (and a fixed area ratio of 0.5 ) and one for the $\mathrm{SiO}_{2}$ signal at about $104.3 \mathrm{eV}$ binding energy. Shirley background subtraction between 96 and $109 \mathrm{eV}$ and Gauss-Lorentz shaped peaks were used. The presence of $\mathrm{Si}_{2} \mathrm{O}_{3}, \mathrm{SiO}$ and $\mathrm{Si}_{2} \mathrm{O}$ chemical states at the interface was neglected. The $\mathrm{SiO}_{2}$ oxide layer thickness was calculated using Eqn. (1) and values given in ref. [4].

$$
\begin{aligned}
& \text { Oxide thickness }=E A L \cdot \cos (\Theta) \text {. } \\
& \ln \left(\frac{\text { area }(\text { film })}{\text { area }(\text { substrate })} \cdot \frac{\text { area }(\text { Si reference })}{\text { area }\left(\mathrm{SiO}_{2} \text { reference }\right)}+1\right)
\end{aligned}
$$




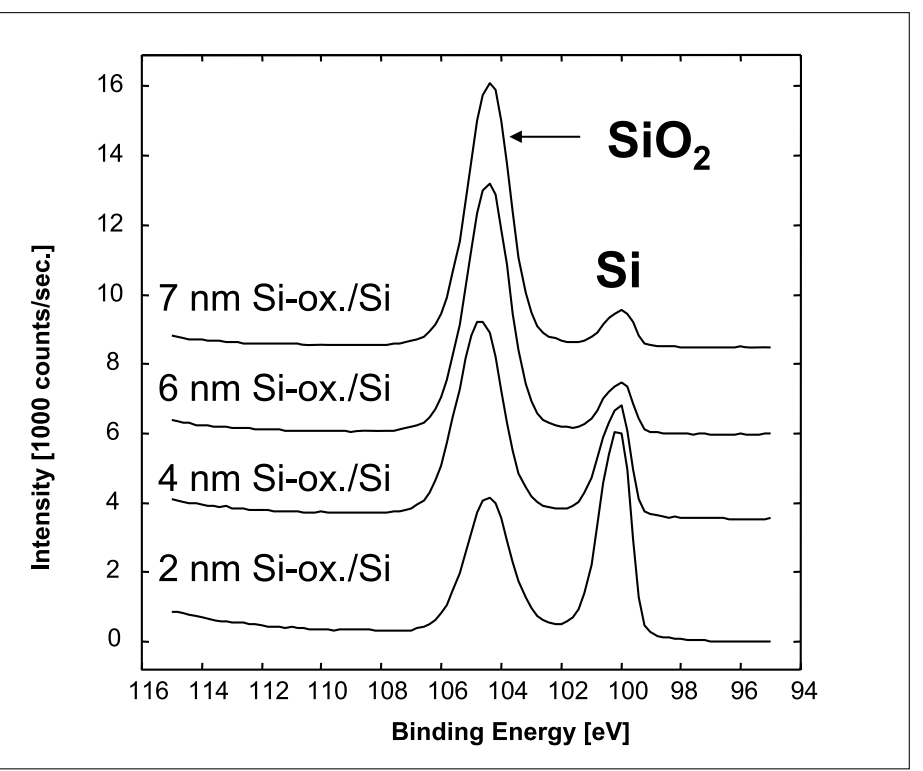

Fig. 2. Si $2 p$ photoelectron signal from different silicon oxide layers on $\mathrm{Si}(111)$. The attenuation of the Si substrate signal by the increasing oxide thickness can clearly be seen.

The Si wafer samples with nominally 2, 4, 6 and $7 \mathrm{~nm}$ thick thermal oxide layers were provided by the National Physical Laboratory, Teddington, UK, for an international comparison on thickness measurements for ultrathin $\mathrm{SiO}_{2}$ layers on $\mathrm{Si}$. The oxide layers were grown by thermal oxidation in furnaces designed for ultrathin gate oxides in semiconductor manufacturing. The nominal oxide layer was determined using a Philips PZ 2000 ellipsometer designed for production line thickness determination. This instrument provided maps with a precision of $\pm 0.002 \mathrm{~nm}$, allowing samples to be selected from regions that were homogeneous to $1 \%$.

The values obtained by XPS analysis for the nominally 2, 4, 6 and $7 \mathrm{~nm}$ thick thermal oxide layers are $1.81,3.44,5.52$, and 6.94 $\mathrm{nm}$. The values obtained are systematically too low which is also caused by neglecting the presence of $\mathrm{Si}_{2} \mathrm{O}_{3}, \mathrm{SiO}$ and $\mathrm{Si}_{2} \mathrm{O}$ chemical states at the interface. So the thickness measured rather represents the thickness of the $\mathrm{SiO}_{2}$ layer and not the total oxide layer thickness. For a very detailed data analysis including the interface oxides, see [4].

\subsection{AES}

AES is similar to XPS, however, in AES the surface atoms are excited by an electron beam in the range of 3-50 kV. The incoming electron can remove a core electron leaving an unoccupied core electron state. This empty core state is then filled by an outer shell electron dropping to the lower energy orbital. The energy associated with this transition must be released either by the emission of an X-ray photon or by transferring the energy to another outer shell electron, which then is ejected from the atom and is called an Auger electron after the
French scientist Pierre Auger. In an Auger electron spectrum the energy of the emitted electrons is determined with an electron energy analyzer. The surface sensitivity is analogous to XPS in the range of 1-5 nm. From the intensity of the Auger signals, the elemental concentration at the surface can be determined. Also, Auger electrons contain some chemical information about the atom they have been emitted from. Since three different electron orbitals are involved in the Auger process, the chemical information is usually contained in a characteristic peak shape with several additional small peaks. The determination of the chemical state is usually done by comparison with a reference spectrum. The detection limit is dependent on the elements and the cross section of the different transitions in the Auger process and is between 0.5 and 2 atomic percent. All elements can be measured except hydrogen and helium.

The quantitative determination of the elemental concentrations is slightly disturbed by the chemistry of the atoms involved since the three electron orbitals involved, as well as the cross sections for the different transitions, are influenced by the chemical situation. Additionally, one or two electron levels involved may be in the valence band, which is severely different for different chemical species. Therefore, the accuracy of the quantitative concentration obtained is, without additional reference measurements, only about $20-30 \%$. Since in AES the surface is excited by an electron beam, usually only electrical conductive materials can be analyzed, however, the electron beam can be focused to a small spot allowing a lateral resolution below $10 \mathrm{~nm}$. When the electron beam can be scanned, allowing the acquisition of elemental or even chemical surface maps, these analytical instruments are also called scanning Auger microscope (SAM). Below, an example of AES analysis is presented.

The lateral resolution of scanning Auger microscopy can be seen in Fig. 3. The GaAs/AlAs super lattice consists of a multi-layered structure with four GaAs layers and five AlAs layers, with each layer 10 $\mathrm{nm}$ thick. The cross section of the sample was analyzed in a PHI 700 Scanning Auger Nanoprobe. The Ga LMM Auger peak was acquired in each single pixel of Fig. 3 and the Ga intensity is displayed as the brightness of each pixel. The Ga map was collected for 200 min at an electron beam setting of $20 \mathrm{kV}$ and $2 \mathrm{nA}$ and shows the excellent Auger spatial resolution and the long term stability during extended acquisitions.

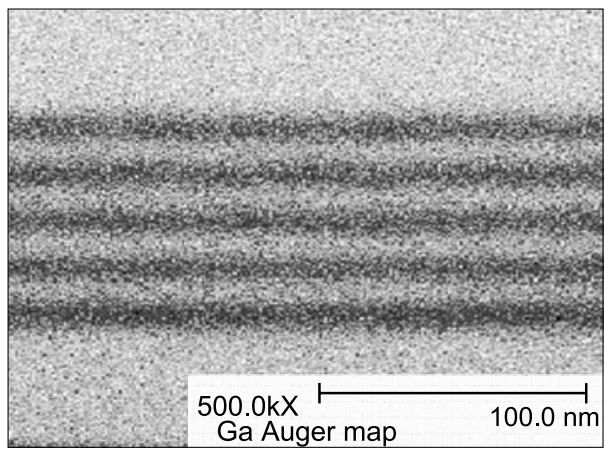

Fig. 3. Ga Auger signal intensity map of a GaAs/ AlAs super lattice structure with each layer $10 \mathrm{~nm}$ thick (picture from Physical Electronics, Chanhassen, MN, USA)

\subsection{ToF-SIMS}

The limitations of electron probe X-ray and Auger spectroscopy in the characterization of complex organic thin films is to a certain extent complemented by time-offlight secondary ion mass spectrometry (ToF-SIMS). The use of energetic focused ion beams to generate secondary particles by bombardment of a sample surface with energetic projectiles (typically atomic or cluster species) in the range of about 5-25 $\mathrm{keV}$ was described in detail some 20 years ago [5]. The energy that is deposited by the primary ions in the surface region of a material produces a collision cascade. This results in the ejection of a wide range of secondary electrons, positively and negatively charged atoms and molecular fragments (fraction: $10^{-6}-10^{-1}$ ), and neutral particles. The latter amount to up to $90 \%$ of all species produced, depending on the chemical composition of the surface. The secondary ion yield usually increases for larger projectiles, but also comes with increased damage to the surface. This results in extensive fragmentation and bond braking processes near the collision site. Therefore, atomic species and small, unspecific low-mass fragments arise from this part of the surface. Large, 
compound specific molecular fragments are produced as the result of less energetic collisions at locations away from the starting point of the collision cascade, taking place in the top 2-3 layers of the sample. This range also defines the analytical sensitivity of the Tof-SIMS technology.

In principle, SIMS is a destructive process that is often used to obtain depth profiles and quantitative data about trace element impurities on semiconductor and metal surfaces. The poor duty cycle of pulsed primary gun operation usually restricts recording of depth profiles to very shallow structures like gate oxide ion implant systems. However, by restricting the primary ion dose to values $\leq 10^{13}$ ions $\mathrm{cm}^{2}$, so-called static SIMS (SSIMS) conditions can be achieved. In this mode of operation, only about $1 \%$ of all species located within the analysis volume will be consumed during the measurement, leaving the surface almost destruction-free and therefore accessible to further investigations.

Due to the small amount of secondary ions that are generated during each primary ion pulse, a mass analyzer system with a ultra-high overall transmission probability is required for SSIMS. Time-of-flight (ToF) techniques have been recognized to offer the unique properties of high sensitivity, excellent mass resolution, wide mass range and accurate mass determination in combination with multiplexed detection of all fragments for most effective duty cycles [6][7]. In order to achieve high mass resolution modern ToF-SIMS instruments are equipped with a reflectron type time-of-flight mass analyzing system. The field-free drift region of $2 \mathrm{~m}$ is roughly split into two parts of equal length. A precisely spaced array of electrostatic lenses with gradually increasing retarding potentials is located at the turning point and acts as an ion mirror. This set-up actively compensates for the energy distribution of the secondary particles, which would otherwise degrade the mass resolution of the instrument. Time-of-flight mass spectra are recorded by accurately controlling the time intervals between source pulsing and detection of the secondary ions via single ion counting in a time-to-digital converter. With this instrumental set-up, a mass resolution $\mathrm{M} / \Delta \mathrm{M} \geq 7500$ at $\mathrm{m} / \mathrm{z}=28$ can routinely be achieved in positive and negative ToF-SIMS spectra using a $\mathrm{Cs}^{+}$or cluster primary ion gun.

The basic SIMS equation to describe the intensity of the secondary ion current, $\mathrm{I}_{\mathrm{s}}^{\mathrm{m}}$ of species $\mathrm{m}$ is:

$\mathrm{I}_{\mathrm{s}}^{\mathrm{m}}=\mathrm{I}_{\mathrm{p}} \mathrm{y}_{\mathrm{m}} \alpha^{+} \theta_{\mathrm{m}} \eta$

$\mathrm{I}_{\mathrm{p}}$ is the primary ions flux, $\mathrm{y}_{\mathrm{m}}$ is the sputter yield, $\alpha^{+}$is the ionization probability to positive ions, $\theta_{\mathrm{m}}$ is the fractional concentration of $\mathrm{m}$ in the surface layer and $\eta$ is the transmission of the analysis system. Several instrumental innovations have been introduced over the past decade to maximize $\mathrm{I}_{\mathrm{s}}^{\mathrm{m}}$ and, therefore, offer the possibility to obtain as much signal as possible as a result of a single primary ion collision event.

The development of polyatomic or cluster primary ion sources for SSIMS applications, which produce an increased secondary ion yield, now offers the possibility to analyze relatively small domains of a surface while maintaining the necessary mass resolution for chemical assignment of large molecular fragments, i.e. record high mass resolution surface chemical images. This feature is of particular importance for the characterization of very small domains where excessive bombardment with high energy projectiles produces surface damage within the time frame necessary to record and detect enough secondary particles for unambiguous interpretation of surface chemistry. With increasing number of applications of ToF-SIMS to the field of surface and interface related biological phenomena, the number of high-mass secondary ions generated per impinging primary ion is a critical value for the application of TOF-SIMS spectrometry to such problems. The first type of cluster ion source producing $\mathrm{SF}_{6}{ }^{+}$primary ions was developed more than a decade ago, but lacked the necessary beam intensity and focus properties for widespread commercial applications. With the introduction of gold $\left(\mathrm{Au}^{+}, \mathrm{Au}_{2}{ }^{+}\right.$ and $\left.\mathrm{Au}_{3}{ }^{+}\right)$and bismuth $\left(\mathrm{Bi}_{\mathrm{n}}{ }^{+}\right)$cluster ion sources to the market [8], previous limitations in operational performance could be overcome and high yield fine focus primary ion beams became available. While the ion yield from molecular ion beams $\left(e . g . \mathrm{Au}_{3}{ }^{+}\right)$ is at least a factor of 100 higher than from mono-atomic primary ion beams $\left(e . g . \mathrm{Cs}^{+}\right.$ or $\mathrm{Ga}^{+}$) [9][10], it has sometimes also been reported in the literature that the so-called damage rate is not significantly higher. This value, often referred to as the disappearance cross-section, will ultimately determine how much of the increased ion yield will finally translate into increased total signal.

In the following, we present examples to show the potential of ToF-SIMS for the chemical characterization of structured surfaces with thin overlayers.

\subsubsection{Electrochemically Stimulated Desorption of Poly(L-Lysine)-graft- Poly(Ethylene Glycol) (PLL-g-PEG) Adlayers from a Patterned Titanium/ Silicon Oxide Surface}

Poly(L-lysine)-g-poly(ethylene glycol) (PLL-g-PEG), a highly protein-resistant material, was uniformly adsorbed from solution onto a patterned substrate with conducting and insulating stripes of about $15 \mu \mathrm{m}$ width and subsequently subjected to a direct current (dc) voltage. Under the influence of an ascending cathodic and anodic potential, there was a steady and gradual loss of PLL-g-PEG from the conductive titanium surface areas while no desorption was observed on the insulating silicon-oxide stripes. This difference was used for the study of the electrochemical response of PLL-g-PEG as a tool to fabricate an experimental platform for biorecognition studies based on the controlled surface functionalization of the titanium areas while maintaining a protein-resistant background on the silicon oxide regions [11]. The surface modified substrate was then subjected to $+1800 \mathrm{mV}$ (referenced to the silver electrode). It was observed that the PLL-g-PEG film was removed from the titanium stripes without inducing any polyelectrolyte loss from the silicon oxide regions. The localized release of the protein-resistant polymer by an electric field from conducting surface domains was observed to be well-defined within a resolution of up to $2 \mu \mathrm{m}$. Imaging ToF-SIMS is highly suitable for analyzing patterned surfaces with thin molecular adlayers due to the very shallow information depth and the good lateral resolution.

Fig. 4a. shows the total ion ToF-SIMS image of a surface modified titanium/silicon oxide substrate in the mass range of $\mathrm{m} / \mathrm{z}=1$ to $\mathrm{m} / \mathrm{z}=100$. The difference between the intensities on the alternating $15 \mu \mathrm{m}$ titanium and $15 \mu \mathrm{m}$ silicon oxide stripes was due to the uneven charge distribution on the heterogeneous microstructures during the ToF-SIMS measurements. However, the well-defined border between the conducting titanium $(\mathrm{m} / \mathrm{z}=48)$ and insulating silicon oxide $(\mathrm{m} / \mathrm{z}=28)$ regions on the micron dimension shows the analytical potential of the method for detecting chemical differences on this length scale. The effect is clearly visualized in the grey-scale Fig. 4b and Fig. 4c. Brighter domains indicate a higher abundance of the respective mass. ToF-SIMS imaging also revealed the presence of a characteristic PEG molecular species $\left(\mathrm{m} / \mathrm{z}=45, \mathrm{C}_{2} \mathrm{H}_{5} \mathrm{O}^{+}\right)$which was found on the silicon oxide region but not on the titanium stripes (Fig. 4d) after unspecific adsorption of PLL-g-PEG on the patterned substrate followed by the application of $+1800 \mathrm{mV}$ to the conducting parts of the sample. The distribution image of characteristic molecular fragments of the polymer film produced a high contrast with much more signal intensity on the silicon oxide stripes. This clearly indicates the loss of the monolayer from the titanium areas upon the anodic polarization at $+1800 \mathrm{mV}$ as the result of electrochemically induced PLL-g-PEG desorption from the conductive titanium surface without significantly 

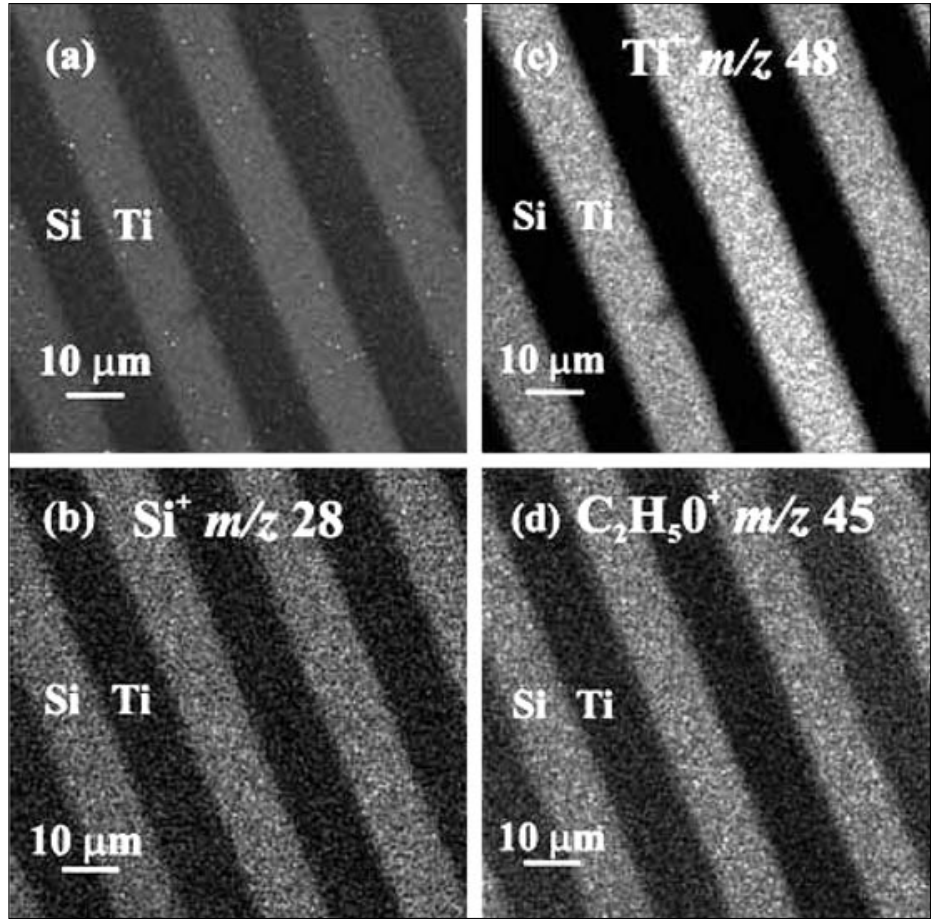

Fig. 4. Positive-ion ToF-SIMS images (positive secondary ions) indicated the presence of PLL-g-PEG layers on a substrate with $15 \mu \mathrm{m}$ titanium/15 $\mu \mathrm{m}$ silicon oxide stripe after electrical polarization to $+1800 \mathrm{mV}$ for $60 \mathrm{~min}$ : (a) Total secondary ion intensity between masses $\mathrm{m} / \mathrm{z}=1$ and $\mathrm{m} / \mathrm{z}=100$ (b) Si ${ }^{+}$ $(\mathrm{m} / \mathrm{z}=28)$ intensity (c) $\mathrm{Ti}^{+}(\mathrm{m} / \mathrm{z}=48)$ intensity (d) Most prominent molecular species $\mathrm{C}_{2} \mathrm{H}_{5} \mathrm{O}^{+}(\mathrm{m} / \mathrm{z}=45)$ characteristic for PEG indicate that the PLL-gPEG adlayer was almost completely removed from the titanium regions but remained adsorbed on the silicon coated stripes.

affecting the polyelectrolyte layer on the silicon region.

\section{Summary}

We aimed in this publication to present very briefly the most important analytical techniques available today for the characterization of surface chemical related phenomena with their individual advantages and limitations. No such contribution can attempt to cover the full variety of possible applications, nor discuss all instrumental innovations. However, the limited space available to present XPS, AES, and TOFSIMS methods should provide the reader with a basic idea of the analytical potential of these techniques.

While XPS and AES can provide quantitative data of the outermost surface region of a material, and, therefore, are indispensable for detecting the extent and quality of surface related phenomena, the combination of high mass resolution, ultra high sensitivity and spatial resolution in static ToF-SIMS (SSIMS) generate an enormous amount of information about the chemical composition of the topmost surface layers. Parallel application of the techniques sets almost no restrictions to the type of material under investigation, and provides the analyst with the necessary tools to obtain a complete picture of the chemical state of a surface.

However, future advances of the techniques depend on the development of more advanced data analysis schemes to assist the experimentalist to utilize all information in a spectrum or image.

The most promising applications of XPS and ToF-SIMS imaging with mono- chromatic X-ray sources and state-of-theart cluster ion beam technology will be the determination of complex high-mass biologically active molecules in two- and three dimensions. The first application of $\mathrm{Au}_{\mathrm{n}}{ }^{+}$and $\mathrm{C}_{60}{ }^{+}$primary ion sources to such applications have recently been published [8][12].

\section{Acknowledgement}

Special thanks are due to Dr. Clarence Tang for recording TOF-SIMS spectra and to Günther Hobi for solving many technical problems. The authors also wish to thank the management of Empa for continuous financial support.

Received: August 29, 2006

[1] P. Bernhard, J. Maul, U. Ott, C. Sudek, M. Escher, N. Weber, M. Merkel, B. Krömker, D. Funnemann, G. Schönhense, Nucl. Instr. and Meth. in Phys. Res. 2006, B 246 , 275-280.

[2] D. Briggs, M.P. Seah, 'Practical Surface Analysis', John Wiley \& Sons, Chichester, 1990

[3] C.J. Powell, A. Jablonski, J. Electron Spectrosc. Relat. Phenom. 2001, 114-116, 1139-1143.

[4] M.P. Seah et al., Surf. Interface Anal. 2004, 36, 1269-1303.

[5] A. Benninghoven, F.G. Ruedenauer, H.W Werner, 'Secondary Ion Mass Spectrometry', John Wiley \& Sons, New York, 1987.

[6] J.C. Vickerman, A.J. Swift, in 'Surface Analysis - The Principal Techniques', Ed. J.C. Vickerman, John Wiley \& Sons, Chichester, 1997, p. 135.

[7] R.J. Cotter, J. Anal. Chem. 1992, 64, 1027A.

[8] N. Winograd, Anal. Chem. 2005, 77, A143-A149.

[9] S.C.C. Wong, R. Hill, P. Blenkinsopp, N.P. Lockyer, D.E. Weibel, J.C. Vickerman, Appl. Surf. Sci. 2003, 203, 219-222.

[10] D.E. Weibel, N. Lockyer, J.C. Vickerman, Appl. Surf. Sci. 2004, 231-232, 146-152.

[11] C.S. Tang, P. Schmutz, S. Peronis, M. Textor, B. Keller, J. Vörös, Biotechnol. Bioeng. 2005, 91, 285-295.

[12] P. Sjoevall, J. Lausmaa, B. Johansson, Anal. Chem. 2004, 76, 4271-4278. 